\title{
Fractura transdiscal y subluxación C5-C6 en paciente con espondilitis anquilo- poyética
}

\author{
A. Carrillo-Colmenero ; T. García-de la Oliva y H.F. Cabrera Ortiz*
}

Servicios de Radiodiagnóstico y de Neurocirugía*. Hospital Regional Universitario Carlos Haya. Málaga.

\section{Resumen}

La fractura cervical es una posible complicación, no infrecuente, de la espondilitis anquilopoyética (EA) de larga evolución.

La actual nota clínica recoge un caso registrado en nuestro hospital, consistente en una fractura transdiscal y luxación C5-C6, con paraplejia final residual.

Su gravedad, pues, radica en su importante morbimortalidad en este tipo de pacientes.

El presente artículo centra su atención sobre el diagnóstico precoz, que posibilitará un manejo terapéutico personalizado, y sobre la prevención, en la medida de lo posible, de esta grave complicación.

Analizamos el contexto clínico del paciente, los factores predisponentes, el mecanismo lesional, la expresión radiológica, las secuelas neurológicas y el manejo terapéutico.

PALABRAS CLAVE: Espondilitis anquilopoyética. Fractura cervical. Lesión neurológica.

C5-C6 transdiscal fracture and subluxation in a patient with ankylosing spondylitis

\section{Summary}

Cervical fracture is not a rare complication of longterm ankylosing spondylitis (AS). We are reporting a case of cervical fracture in a patient affected by which was complicated by a transdiscal fracture, C5-C6 subluxation, and residual paraplegia.

We analyse clinical presentation, predisponent factors, injury mechanism, radiologic features, neurological deficits, and terapeutic management.

We discuss the accurate diagnosis and the most suitable treatment for this serious complication, as well as prevention measures.

Recibido: 01-10-04. Aceptado: 07-03-05
KEY WORDS: Ankylosing spondylitis. Cervical fracture. Cervical subluxation.

\section{Introducción}

La prevalencia de la EA (espondilitis anquilopoyética) asociada a la presencia de antígeno HLA B27 y a sacroileítis oscila en torno al $0,1 \%$ en la población general ${ }^{9}$.

La columna anquilosada es muy susceptible a las fractu$\operatorname{ras}^{10}$, por la progresiva pérdida de movilidad y osteoporosis secundaria; sin embargo, no se ha logrado establecer correlación entre densidad mineral ósea y fracturas vertebrales en estos pacientes ${ }^{14}$.

La incidencia de fractura vertebral en pacientes con EA es cuatro veces mayor que en la población general ${ }^{9}$.

Las fracturas suelen ser provocadas por un traumatismo menor, como caídas ${ }^{17.4}$ estando de pie o caminando (el mecanismo más común) o por accidente de tráfico (el segundo más frecuente).

El 75\% de las fracturas ocurre a nivel de la columna cervical, especialmente en la unión cervicotorácica C5-T1 $1^{4.10 .15}$.

En este artículo, se pretende analizar un caso de fractura vertebral en un paciente diagnosticado de EA, con el fin de definir mejor los factores predisponentes, el mecanismo lesional, las características radiológicas, el déficit neurológico asociado y las estrategias terapéuticas.

\section{Caso clínico}

Se trata de un varón de 59 años de edad en cuyos antecedentes personales destacan: hipercolesterolemia, cardiopatía isquémica (infarto agudo de miocardio en Junio del 2000 y by-pass aortocoronario) y espondilitis anquilopoyética de larga evolución El paciente tras una caída accidental, sufre traumatismo craneoencefálico con flexoextensión del cuello, presentando cervicobraquialgia, parestesias y

Abreviaturas. AS: ankylosing spondylitis. EA: espondilitis anquilopoyética. RM: resonancia magnética nuclear. STIR: secuencias con supresión grasa o short-time inversion recovery. TAC: tomografia axial computarizada. 


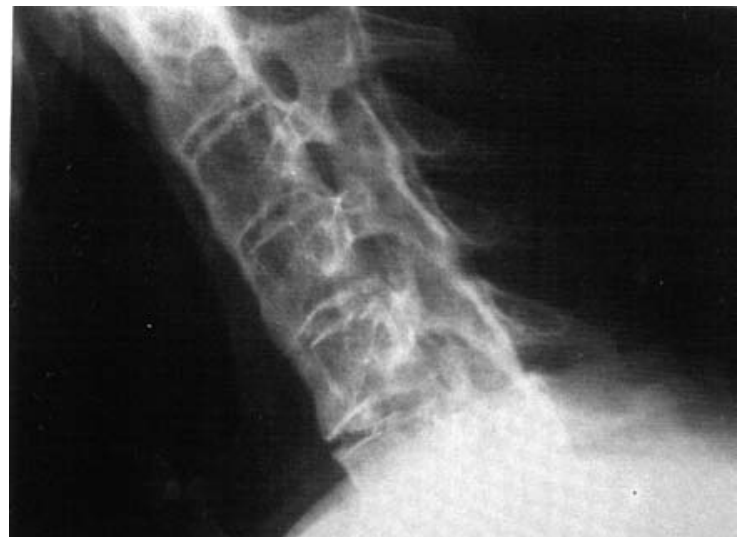

Figura 1. Radiografía lateral de columna cervical evidenciando rectificación cervical, calcificación ligamentosa y disrupción del ligamento longitudinal anterior en C5-C6.

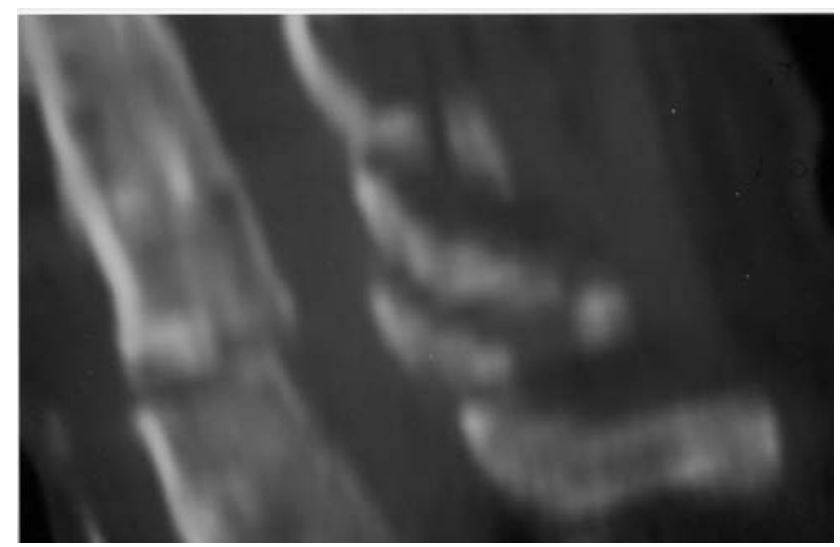

Figura 2. Reconstrucción sagital de TAC de columna cervical mostrando la fractura transdiscal y de elementos posteriores a nivel de C5-C6.

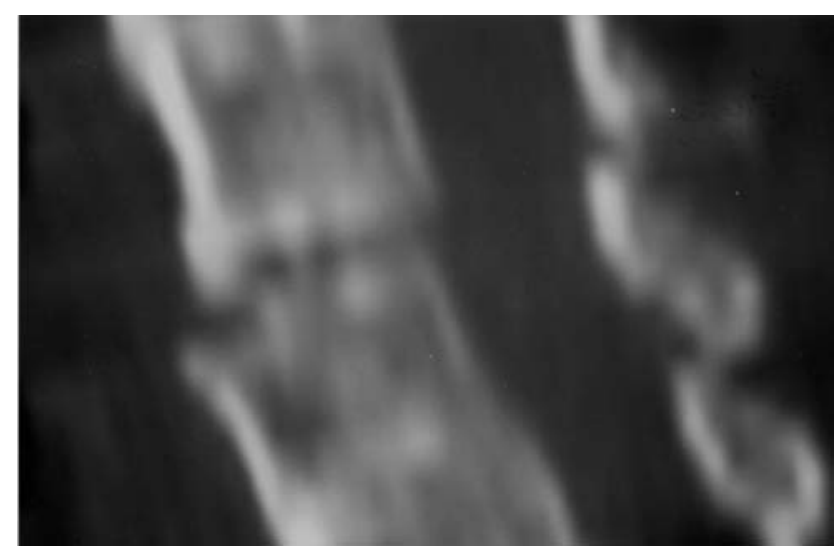

Figura 3. Corte axial de TAC de columna cervical mostrando la fractura de elementos posteriores.

disminución de fuerzas a nivel de miembros superiores y paraplejía.

El paciente ingresó en el Servicio de Neurocirugía con escala de Glasgow 15, nivel de sensibilidad cinco centímetros por debajo de la línea intermamilar, hipoestesia y fuerza $2 / 5$ en miembros superiores y ausencia de fuerza y de sensibilidad en miembros inferiores.

Las radiografías de columna cervical realizadas de urgencia (Fig. 1) evidenciaban una marcada rectificación cervical, calcificación de ligamentos de sostén intervertebrales, tanto anteriores como posteriores, y disrupción del ligamento longitudinal anterior entre C5 y C6.

Posteriormente la TAC (tomografía axial computarizada) de columna cervical (Figs. 2 y 3) mostró la fractura transdiscal entre C5 y C6 y de elementos posteriores, con rotura de ligamentos longitudinal anterior y posterior, y luxación anterior del bloque vertebral inferior.

En la RM cervical inicial se objetivó una fracturaluxación C5-C6, con anterolistesis (Figs. 4, 5 y 6)). Existía alteración de la señal intramedular en el nivel patológico, correspondiente a edema más hemorragia, e impronta posterior medular de elementos posteriores y anterior del extremo posteroinferior de C5 (Figs 5 y 6). Era visible un sangrado en el espacio intervertebral C5-C6, en el lugar teórico del platillo vertebral superior del cuerpo de C6, en relación con el probable mecanismo de cizallamiento. El disco C5-C6 permanecía por encima de la hemorragia sin alteración de señal y presentaba un prolapso concéntrico (Fig. 7).

En el lapso temporal desde la realización de las radiografías cervicales hasta la de la TAC y RM de urgencia objetivamos cómo la inicial fractura transdiscal y de elementos posteriores (esta última no visible en radiografías simples) se complica con luxación C5-C6.

Bajo anestesia general se realizó artrodesis anterior C5C6 con discectomía, se interpuso injerto óseo heterólogo y se fijó con placa y tornillos. La calcificación completa del disco intervertebral requirió la ayuda de un "drum" para retirarlo al completo. A continuación la cirugía fue complementada con un "halo vest" cráneo-torácico.

La RM post-quirúrgica evidenció la fijación anterior con tornillos en los cuerpos vertebrales C5-C6. Asímismo existía un aumento de señal intramedular en T1 (Fig. 8) y más intenso en T2 (Fig. 9) y STIR (fig. 10), en relación con el foco hemorrágico edematoso subagudo intramedular, y otro foco lesional localizado a nivel de elementos posteriores.

Actualmente, el paciente, desde la caída y tras dos meses de seguimiento, permanece clínica y neurológicamente con paraplejía residual en un centro rehabilitador.

\section{Discusión}

El caso referido afecta a un varón, al igual que la mayoría de casos descritos en la literatura ${ }^{7,10,12,20,22}$. La gravedad del traumatismo se enfatiza por el hecho de que, en su 


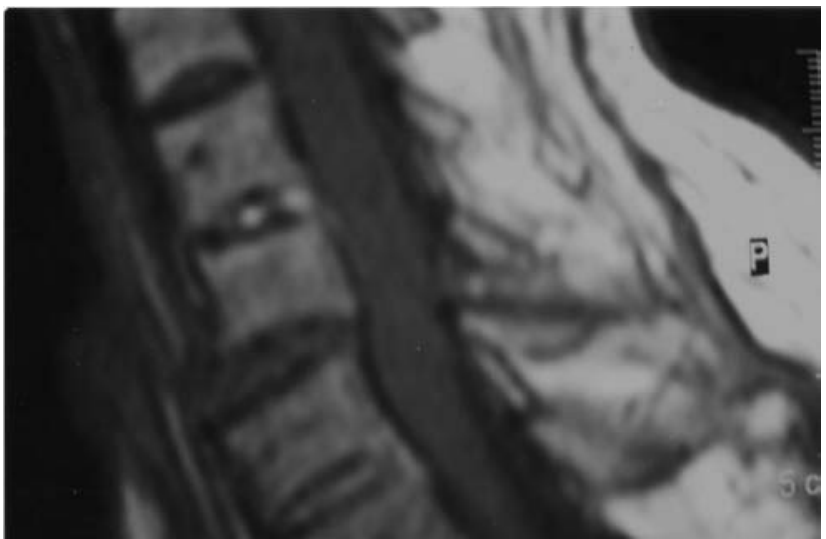

Figura 4. Secuencia $T 1$ (reconstrucción sagital) de la RM inicial de columna cervical mostrando fractura luxación C5-C6, con anterolistesis

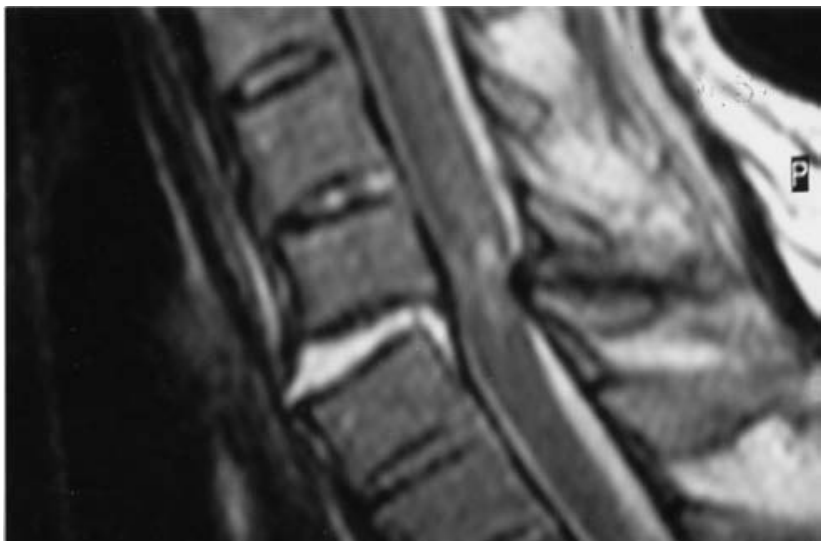

Figura 5. Secuencia T2 (reconstrucción sagital) de la RM inicial, mostrando aumento de señal intramedular, consecuencia del edema y hemorragia, más impronta posterior medular de elementos posteriores.

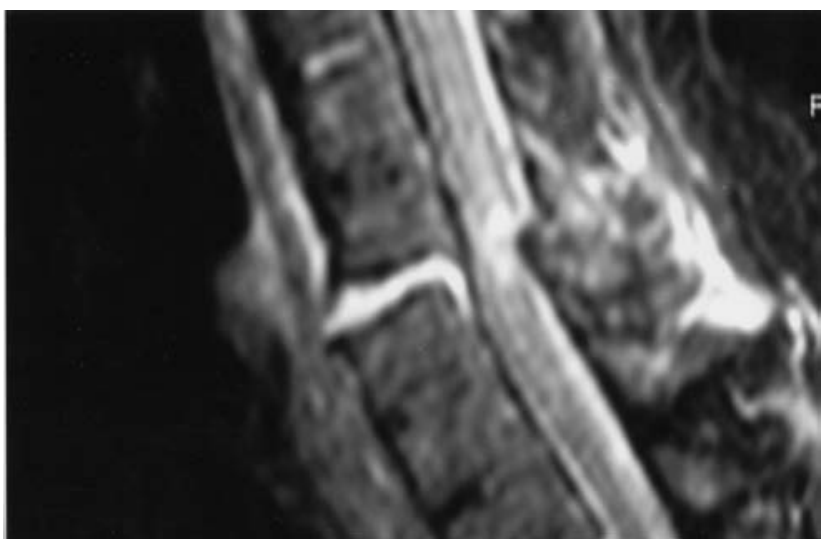

Figura 6: Secuencia STIR (reconstrucción sagital) de la RM inicial mostrando alteración de la señal intramedular, transdiscal y a nivel de los elementos posteriores de C5 y C6.

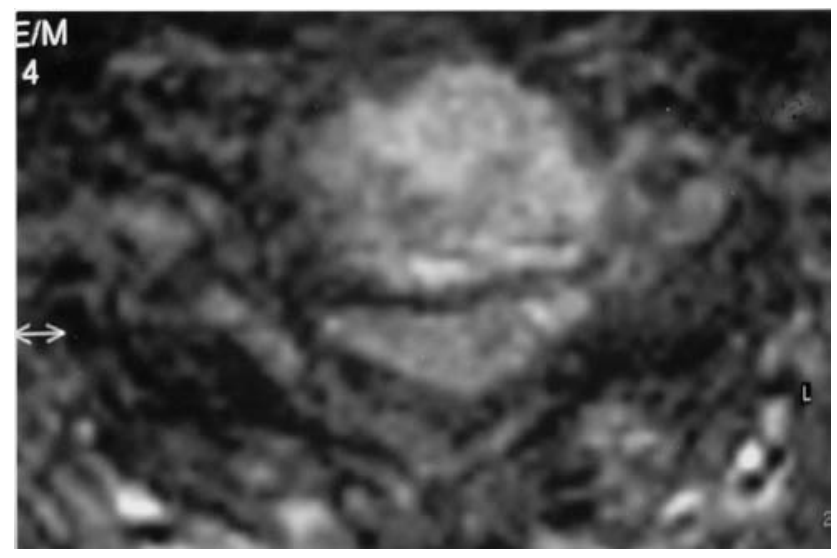

Figura 7. Secuencia $T 1$ (reconstrucción axial) de la RM inicial mostrando prolapso concéntrico discal C5-C6.

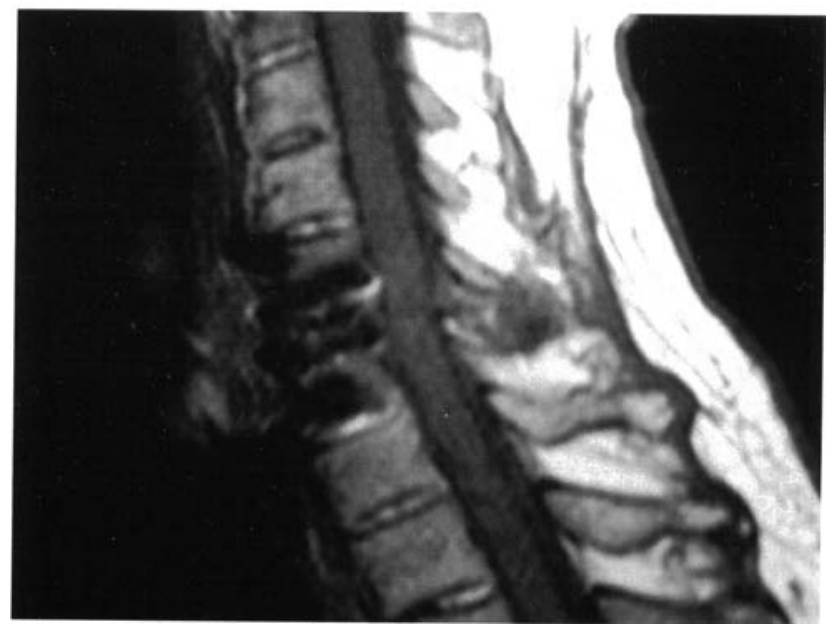

Figura 8. Secuencia $T 1$ (reconstrucción sagital) de la RM postquirúrgica mostrando la fijación anterior y un discreto aumento de la señal intramedular.

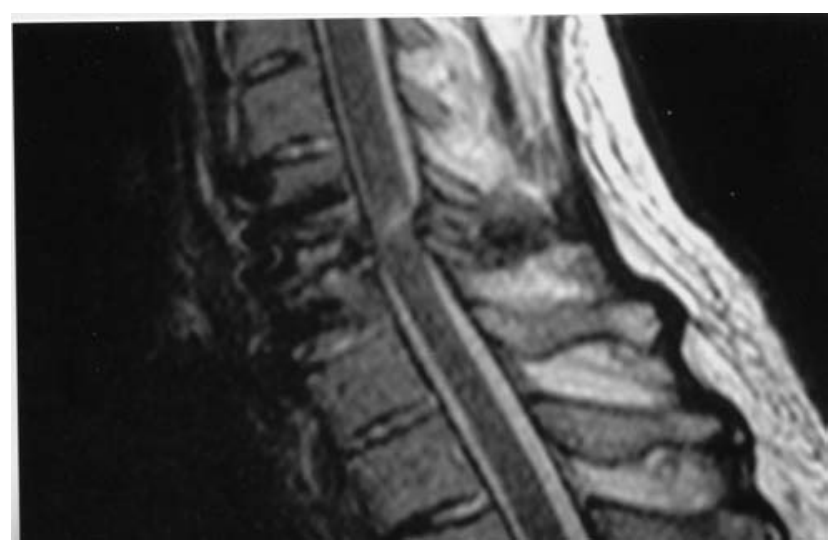

Figura 9. Secuencia T2 (reconstrucción sagital) de la RM postquirúrgica mostrando aumento de señal intramedular consecuencia del foco hemorrágico-edematoso subagudo. 


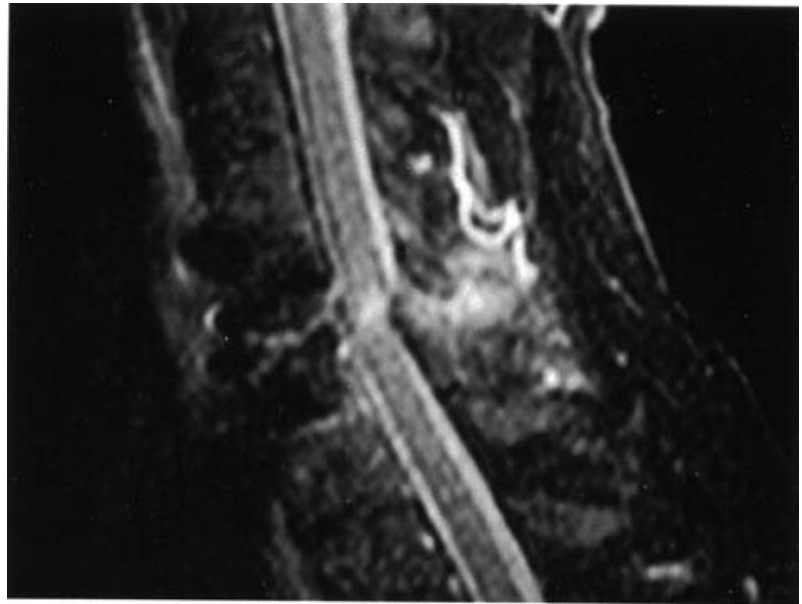

Figura 10. Secuencia STIR (reconstruccion sagital) de la RM postquirúrgica mostrando aumento de señal residual a nivel medular y de elementos posteriores.

mayoría, son traumatismos mínimos ${ }^{9,21}$. Así mismo, la gravedad de la fractura se refleja en las secuelas neurológicas; la incidencia de déficit neurológico oscila entre 40 y $70 \%$, y la tasa de mortalidad es del $35 \%$, según refleja la literatura revisada ${ }^{10,20,21,23}$. Es necesario, pues, efectuar en este tipo de pacientes con traumatismo espinal un estudio radiológico de todo el neuroeje, y no sólo de la zona sintomática.

La mayor incidencia de déficits neurológicos secundarios en pacientes con fracturas vertebrales, inicialmente inadvertidas, es de gran importancia en pacientes con $\mathrm{EA}^{2,7,8,13}$, como en el caso que nos ocupa.

El mecanismo lesional más frecuente en este tipo de lesiones es la hiperextensión-distracción (estadío 2 de Allen), produciendo fracturas transdiscales, mientras que el mecanismo flexor suele provocar fracturas a través del cuerpo vertebral (tipo Chance) ${ }^{21,23}$. El paciente en cuestión presentó un mecanismo mixto.

Las alteraciones radiológicas de la luxación en hiperextensión pueden ser muy sutiles o nulas porque la columna vuelve a una alineación casi normal tras el traumatismo.

Esta lesión se sospecha por la existencia de un déficit neurológico grave, a pesar de la escasez de hallazgos radiológicos. Con frecuencia se observa una retrolistesis de vértebra que está por encima de la lesión discal, pero puede ser un grado mínimo ${ }^{18}$.

En los estudios de RM efectuados no se realizaron secuencias postcontraste, aunque sí secuencias de supresión grasa; se ha demostrado que ambas son de gran utilidad en la evaluación del espacio dural y epidural ${ }^{22}$.

La terapéutica de este tipo de fracturas es controvertida $^{6,3}$. La literatura actual promueve tanto medidas conservadoras como quirúrgicas. El manejo conservador incluye situaciones de prolongado reposo en cama con tracción (o collar cervical) y de precoz movilización con halo si la fractura permanece estable. Sin embargo, los propios autores reconocen que la inmovilización sola es difícil y con frecuencia inadecuada, debido a la inestabilidad intrínseca inherente a estas fracturas ${ }^{20}$.

El tratamiento quirúrgico en general se reserva para el manejo de pacientes con déficit neurológico y luxación o afectación de las tres columnas ${ }^{10,19}$, como el caso clínico presentado. Aunque el planteamiento quirúrgico inicial era realizar fijación anterior y posterior, hubo que realizar únicamente fijación anterior por la aparición de insalvables problemas técnicos durante la intervención (a la dificultad inicial para la intubación se sumaron descompensaciones respiratorias por problemas de desintubación con repercusión cardiológico-hemodinámica, en un paciente ya de por sí de alto riesgo).

Reservamos el abordaje posterior para un segundo acto quirúrgico, pero la familia, ante la situación de irreversibilidad y el alto riesgo quirúrgico, decidió posponerla.

La fijación quirúrgica se complementó con una inmovilización externa rígida transitoria para fomentar la fusión ("halo-vest" postquirúrgico) 5,8 .

Finalmente, el paciente fue trasladado de alta de nuestro hospital a un centro rehabilitador.

Como complicación más habitual en la fase aguda postoperatoria destaca la respiratoria; en concreto, el embolismo pulmonar secundario a trombosis venosa profunda, que oscila entre 9 y $90 \%{ }^{1,23}$. En nuestro paciente, con la debida profilaxis (heparina de bajo peso molecular, a razón de $40 \mathrm{U} / 24 \mathrm{~h}$ ), se evitó esta complicación.

Respecto a la prevención, es fundamental la adecuada información al paciente y a sus familiares de la necesidad de evitar caídas y traumatismos. La necesidad de protección cervical en automóviles está muy recomendada en la literatura revisada ${ }^{23}$. También se recomienda la colocación de la cabeza del paciente en posición neutra, con una almohada, ante la sospecha de lesión cervical, durante cualquier movilización (traslado desde el lugar del accidente, realización de pruebas diagnósticas de imagen, etc) ${ }^{16}$.

\section{Conclusión}

Las fracturas cervicales son una complicación posible, no infrecuente, de la EA de larga evolución, y dada su importante morbi-mortalidad en este tipo de pacientes, es fundamental la prevención y el diagnóstico precoz para posibilitar el tratamiento más adecuado en cada caso.

\section{Bibliografía}

1. Alto, S., Pieri, A., D'Andrea, M. et al.: Primary prevention of deep venous trombosis and pulmonary embolism in acute spinal cord injured patients. Spinal Cord 2002; 40: 300303. 
2. Alaranta, H., Luoto, S., Konttinnen, Y.T.: Traumatic spinal cord injury as a complicacion to ankylosing spondylitis. An extended report. Clin Exp Rheumatol 2002; 20: 66-68.

3. Apple, D.F., Anson, C.: Spinal cord injury occurring in patients with ankylosing spondylitis: a multicenter study. Orthopedics 1995; 18: 1005-1011.

4. Bennett, L., Ohashi, K., El-Khoury, G.: Spondyloarhropatles: ankylosing spondylitis and psoriasic arthritis. Radiol Clin N Am, 2004; 42: 121-134.

5. Detwiler, K.N., Loftus, C.M., Godersky, J.C., Menezes, A.H.: Management of cervical spine injuries in patients with ankylosing spondylitis. J Neurosurg 1990; 72: 210-215.

6. Exner, G., Bötel, U., Kluger, P., Richter, M., Eggers, C., Ruidisch, M.: Treatment of fracture and complication of cervical spine with ankylosing spondylitis. Spinal Cord 1998; 36: 377-379.

7. Finkelstein, J.A., Chapman, J.R., Mirza, S.: Occult vertebral fractures in ankylosyng spondylitis. Spinal Cord 1999; 37: 444-447.

8. Fox, M.W., Onofrio, B.M., Kilgore, J.E.: Neurological complication of ankylosing spondylitis: the intervertebral disc as a cause of cord compression. J Neurosurg 1992; 77: 241246.

9. Hanson, J.A., Mirza, S.: Predisposition for spinal fracture in ankylosing spondylitis. AJR 2000; 174: 150.

10. Hitchon, P.W., From, A.M., Brenton, M.D., Glaser, J.A., Torner, J.C.: Fractures of the thoracolumbar spine complicatiog ankylosing spondylitis. J Neurosurg Spine 2002; 97 : 218-222.

11. Hunter, T., Forster, B., Dvorak, M.: Ankylosed spines are prone to fracture. Can Fam Physician 1995; 41: 12131216.

12. Karasick, D., Schweitzer, M.E., Abidi, N.A., Cotler, J.M.: Fractures of vertebrae with spinal cord injuries in patients with ankylosing spondylitis: Imaging Findings. AJR 1995; 165: 1205-1208.

13. Kauppi, M., Belt, E.A., Soini, 1.: "Bamboo spine" starts to bend-something is wrong. Clin Exp Rheumatol 2000; 18: 513-514.
14. Levine, D.S., Forbat, S.M., Saifuddin, A.: MRI of the axial skeletal manifestations of ankylosing spondylitis. Clin Radiol 2004; 59: 400-413.

15. Mitra, D., Elvins, D.M., Speden, D.J., Collins, A.J.: The prevalence of vertebral fractures in mild ankylosing spondylitis and their relationship to bone mineral density. Rheumatol 2000; 39: 85-89.

16. Moreau, A.P.M., Willcox, N., Brown, M.F.: Immobilisation of spinal fractures in patients with ankylosing spondylitis: Two case reports. Injury 2003; 34: 372-373.

17. Ozgocmen, S., Ardicoglu, O.: Odontoid fracture complicating ankylosing spondylitis. Spinal Cord 2000; 38: 117-119.

8. Resnick, D: Traumatismos físicos. En Huesos y Articulaciones en Imagen. Madrid; Marbán 2001; pp 717-818.

19. Rowed, D.W.: Management of cervical spinal cord injury in ankylosing spondylitis: the intervertebral disc as a cause of cord compresión. J Neurosurg 1992; 77: 241-246.

20. Taggard, D.A., Traynelis, V.C.: Management of cervical spinal fractures in ankylosing spondylitis with posterior fixation. Spine 25; 16: 2035-2039.

21. Ticó, N., Ramón, S., García-Ortun, et al: Traumatic spinal cord injury complicating ankylosing spondylitis. Spinal Cord 1998; 36: 349-352.

22. Ting-Fang Shih, T., Chen, P., Li, Y., Hsu, C.H.: Spinal fractures and pseudoarthrosis complicating ankylosing spondylitis: MRI manifestation and clinical significance. J Comput Assist Tomogr; 25: 164-170.

23. Yilmazlar, S., Kocaeli, H., Doygun, M.: Chance type cervical fracture and neurological deficits in ankylosing spondylitis. Ulus Trauma Derg. 2003; 9: 76-78.

Carrillo-Colmenero, A.; García-de la Oliva, T.; Cabrera Ortiz, H.F.: Fractura transdiscal y subluxación C5-C6 en paciente con espondilitis anquilopoyética. Neurocirugía 2006; 17: 440-444.

Correspondencia postal: A. Carrillo-Colmenero. Servicio de Radiodiagnóstico. Hospital Regional Universitario Carlos Haya. Málaga. 